\title{
Integrating multi-electrode arrays in microfluidic hanging-drop
} networks

\section{Conference Paper}

\section{Author(s):}

Misun, Patrick (D); Shadmani, Amir; Hierlemann, Andreas (i); Frey, Olivier

Publication date:

2017

Permanent link:

https://doi.org/10.3929/ethz-b-000237704

Rights / license:

In Copyright - Non-Commercial Use Permitted

Originally published in:

https://doi.org/10.1109/MEMSYS.2017.7863347

\section{Funding acknowledgement:}

267351 - Seamless Integration of Neurons with CMOS Microelectronics (EC)

296257 - The Body-on-a-Chip (BoC) (EC)

142440 - The Body on a Chip - Human 3-D tissue analogues in microfluidic systems (SNF) 


\title{
INTEGRATING MULTI-ELECTRODE ARRAYS IN MICROFLUIDIC HANGING-DROP NETWORKS
}

\author{
Patrick M. Misun ${ }^{1}$, Amir Shadmani ${ }^{1}$, Andreas Hierlemann ${ }^{1}$ and Olivier Frey ${ }^{1}$ \\ ${ }^{1}$ ETH Zurich, Dept. of Biosystems Science and Engineering, Bio Engineering Laboratory, Basel, \\ Switzerland
}

\begin{abstract}
This work presents a highly versatile and scalable analytical platform for 3D microtissues. The device combines microfluidics for precise control of culture conditions with sensor technology for parallel and real-time monitoring of microtissue metabolism. The technology is based on an inverted, open microfluidic network. Interconnected hanging drops are formed underneath a patterned glass chip, which hosts the integrated microelectrode array. The electrodes can be individually functionalized and used as biosensors. The fabrication is simple, and the overall system can be expanded through the integration of larger numbers of hanging drops and more electrodes. The device can be used as a microfluidic platform for cultivation and continuous monitoring of $3 \mathrm{D}$ microtissues.
\end{abstract}

\section{INTRODUCTION}

\section{Motivation}

Analytical cell culturing systems are important tools in the pharmaceutical industry and basic research. As 3D cell and microtissue cultures have become an important test system, the corresponding platforms and readout methods need to be developed [1]. Suitable culturing systems should host multiple microtissues that can be combined in a fluidic network, which enables continuous medium supply. Perfusion is important to create an environment that better resembles in-vivo conditions, and it enables the application of precise compound exposure protocols.

Microelectrode arrays (MEAs) are versatile analytical tools allowing for parallel detection of multiple analytes at different locations of biological samples. The realization of microelectrode structures in a microfluidic cell-culturing environment is technically challenging because liquid routing and electric circuitry need to be unified in a single "package", while function and connectivity are preserved.

Microfluidic hanging-drop networks were specifically designed to connect different spherical 3D microtissues for long-term cultivation in body-on-a-chip configurations [2]. The first attempts to integrate sensor technologies into hanging-drop networks were realized as hybrid plug-in systems [3]. Here we present a new, more comprehensive technological implementation, in which a multi-electrode array has been directly integrated in a microfluidic hangingdrop network.

\section{Concept}

The device consists of a glass substrate, on which the microelectrode array (MEA) has been patterned (Figure 1a).
The MEA consists of eighteen electrodes that can be functionalized by coating the working electrodes with enzyme-hydrogel layers. The enzyme catalyzes a reaction of, e.g., glucose to produce peroxide that can be amperometrically detected (Figure 1b). The microfluidic hanging-drop network is implemented through microfabricated SU-8 structures patterned directly on top of the array. Medium can then be loaded directly into the structures, confining the liquid. Due to capillary forces, the liquid is dragged through the microfluidic channel system. Hanging drops form below the eight circular regions, which are interconnected through narrow channels (Figure 2).

For read-out, the hanging-drop chip MEA is connected to a CMOS-based multi-potentiostat by inserting it into a card socket on a PCB, which is placed in a cultivation box. The MEA and hanging drops are facing downwards. Tubing is connected to the inlet and outlet ports to enable perfusion.

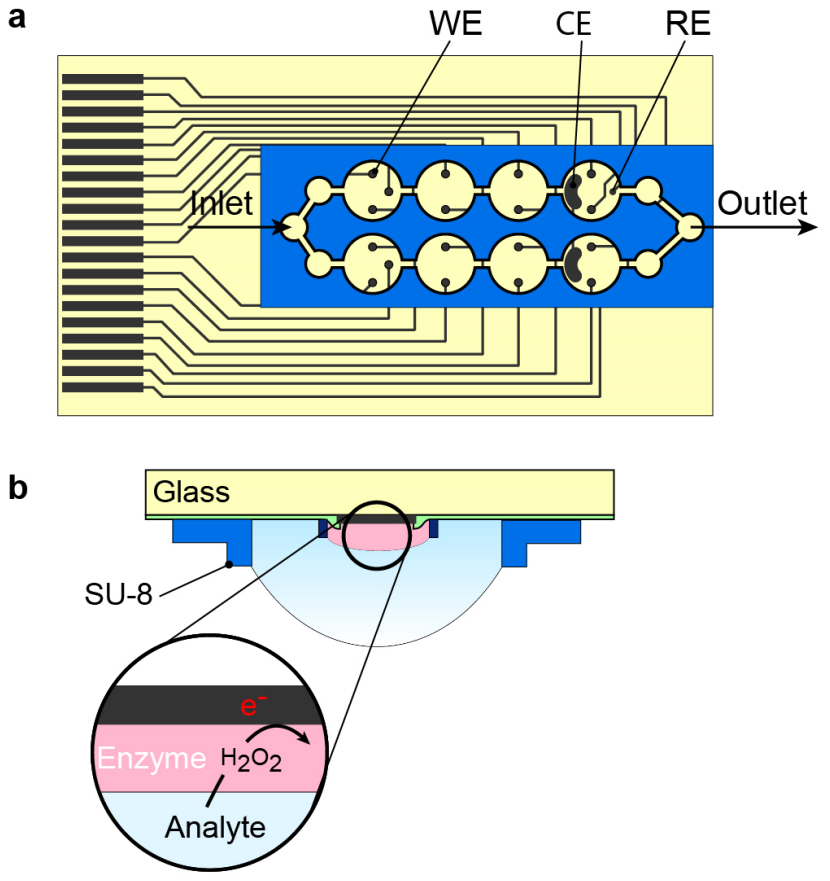

Figure 1: (a) Top view of the microfluidic 2x4-hanging-drop chip. The liquid is guided through an SU-8 structure to form eight hanging drops. The chip features 18 platinum working electrodes (WE), one counter electrode (CE), and one reference electrode (RE). All electrodes are wired to connecting pads on the side of the glass chip. (b) Crosssectional view. The drop formed within the SU-8 rim on a glass substrate. The close-up shows the working principle of the enzyme-based hydrogel coating. 
This novel approach includes both a surface patterned microelectrode array and the means to direct small liquid volumes in a controlled fashion through SU-8 structures, to take advantage of all functions available in microfluidic and sensor technologies. The integration of the electrodes into the microfluidic device and the subsequent functionalization of the biosensors are very simple. The preparation for the measurements, which includes mainly the functionalization of the working electrodes, is performed after all microfabrication process steps have been completed. Due to the completely open chip design, there is no need to close the microfluidic structures after functionalizing the electrodes. Thus, the chip can be rapidly prepared and loaded for measurements, and additionally, the chip can be used multiple times. Moreover, there is no bubble formation, and the gas exchange is continuous. Tissue and cells sediment at the liquid-air interface so any cell or tissue adhesion to surfaces is obviated.

\section{EXPERIMENTAL METHODS}

\section{Device Fabrication}

The device was fabricated on a 4-inch glass wafer using standard photolithography processes. For the fabrication of the metal wires and electrodes, a lift-off resist was first spincoated onto the wafer. A positive photoresist was subsequently deposited on top for the platinum metal patterning. The wafer was UV-exposed through a transparency mask and developed to remove all exposed resist. Platinum was sputtered onto the whole wafer surface, and a lift-off process was subsequently used to remove the metal deposited on the resist while the metal deposited directly on the glass substrate remained. The remaining platinum forms the wires and electrodes of the device. A silicon nitride layer was then deposited through a plasmaenhanced vapor deposition process (PECVD) for passivation of the metal. Another layer of positive photoresist was spin-coated and UV-exposed through a transparency mask, which was used to pattern the openings of the platinum electrodes and connection pads. All the UVexposed regions of the positive resist were removed during a development process. A reactive-ion etching (RIE) fabrication step opened the silicon nitride at the platinum electrode and pad sites. The remaining photoresist was removed by an oxygen plasma process step.

The rim and drop structures of the fluidic network were made of negative SU-8 photoresist. They consist of three layers that were successively deposited onto the glass wafer. Each layer was spin-coated onto the glass wafer with the prefabricated electrodes and UV-exposed through a transparency mask. The first layer, which was $20 \mu \mathrm{m}$ thick, was used to build a small rim around the working electrodes to enhance the hydrogel drop coating of the electrodes during functionalization. The second and third layer, each of which was $250 \mu \mathrm{m}$ thick, were used to structure the fluidic guiding structures.

Finally, the wafer was diced into single hanging-drop chips having a size of $22 \times 40 \mathrm{~mm}^{2}$.

\section{Biosensor functionalization}

The biosensor array features eighteen platinum working electrodes (WE), two counter electrodes (CE), and one $\mathrm{Ag} / \mathrm{AgCl}$ reference electrode (RE). Different coating steps were needed to functionalize each type of electrode.

First, the reference electrode was electrochemically coated with a silver layer, which was later partially transformed into silver chloride to produce a functional $\mathrm{Ag} / \mathrm{AgCl}$ reference electrode.

All the working electrodes were coated with an mphenylenediamine (m-PPD) layer using cyclic voltammetry.

A poly-HEMA based hydrogel was prepared for the selective functionalization of the working electrodes. A precursor solution was mixed from poly(2-hydroxyethyl methacrylate) (pHEMA), the monomer 2-hydroxyethyl methacrylate, (HEMA), tetraethylene glycol dimethacrylate (TEGDMA) as a cross-linker, a photoinitiator, and ethylene glycol as a plasticizer [4]. After this preparation, glucose oxidase (GOx) and catalase enzymes were separately dissolved in DI water and added to the hydrogel precursor solutions. The liquids were mixed well before coating the electrodes with the hydrogel.

A 2- $\mu$ l pipette was used to deposit a small liquid volume of hydrogel solution containing glucose oxidase directly on top of the working electrodes by drop coating. The chip was then UV-exposed for 5 minutes to cross-link the hydrogel membrane. A second layer containing the catalase enzymes was deposited on top and also cross-linked through 5 minutes of UV exposure.

\section{Chip loading and biosensor interface}

The hanging-drop chip was loaded by pipetting medium directly from the top into the drop structures. The liquid then filled the SU-8 rim structures due to capillary forces. Increasing the dispensed volume formed standing drops. A single drop has a total volume of approximately $10 \mu \mathrm{l}$. The loaded chip was then flipped upside down and connected to a CMOS-based multi-potentiostat by plugging it into a card socket on a custom-built PCB, which was placed in a cultivation box (Figure 2a).

The inlets of the hanging-drop chip were connected to syringe pumps via tubing, and the outlet ports were connected to a peristaltic pump to apply controlled perfusion. The device was placed onto a microscope in a cell culture environment at $37^{\circ} \mathrm{C}$ and $5 \% \mathrm{CO}_{2}$. 
a

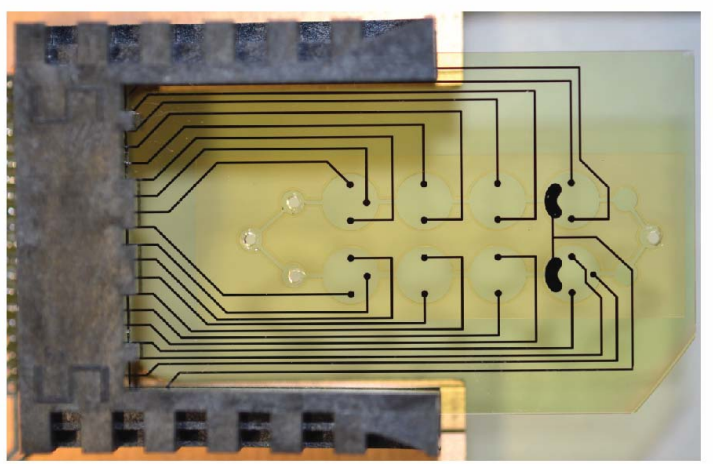

b

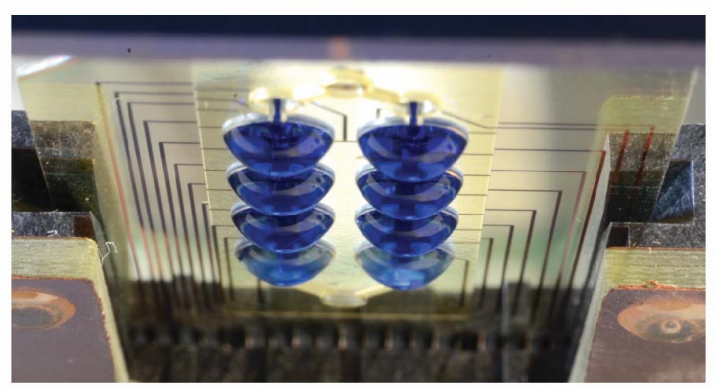

Figure 2: Photographs of the hanging-drop chip illustrating the operation with blue dye. (a) The chip is plugged into a card socket in a custom-built chip-and PCB holder, which is inserted into a cultivation box. This facilitates stable operation and handling of the device. (b) Hanging drops form underneath the circular regions of the $S U-8$ patterned glass substrate.

\section{RESULTS AND DISCUSSION}

The measurements show the characterization and response of the glucose biosensors and the stable operation of the microfluidic device under continuous flow during a calibration procedure. An on-chip calibration curve of the biosensors is shown in Figure 3a. The calibration was performed by subsequently perfusing different glucose concentrations in PBS at a flow rate of $25 \mu 1 / \mathrm{min}$.

Figure 3 illustrates parallel readout from eight hanging drops for a chip with glucose biosensors. Fourteen electrodes (blue) were functionalized with a two-layer GOx/catalase hydrogel membrane. Four selected electrodes (red) were coated with a pure hydrogel layer as a control for the specificity of the recorded signal. Glucose current signals were recorded in real-time. The calibration buffer could be detected on all GOx/catalase functionalized electrodes successively along the flow path. The first signal increase is recorded on the first drop, which is closest to the inlet with a general tubing delay of 2 minutes. The current increase in the following drops is detected with a constant delay of approximately 30 seconds in both drop lines
(Figure 3b). Electrodes coated with pure hydrogel did not show any signal increase.

The noise level and the spatiotemporal response characteristics of the device were recorded at $10 \mathrm{~Hz}$ sampling rate and are shown in Figure $3 \mathrm{~b}$ for four selected biosensor electrodes and one blank electrode as a control along a single drop row. The limit of detection (LoD) of the glucose biosensors is $27 \mu \mathrm{M}$ (three times the background noise at the end of the settling time during the on-chip calibration at $37{ }^{\circ} \mathrm{C}$ under continuous flow). The calibration shows a linear range up to $5 \mathrm{mM}$ glucose. The average sensitivity of the biosensor was determined from fourteen functionalized working electrodes on a single chip and was calculated to be $1.66 \pm 0.33 \mathrm{nA} / \mathrm{mM}$.
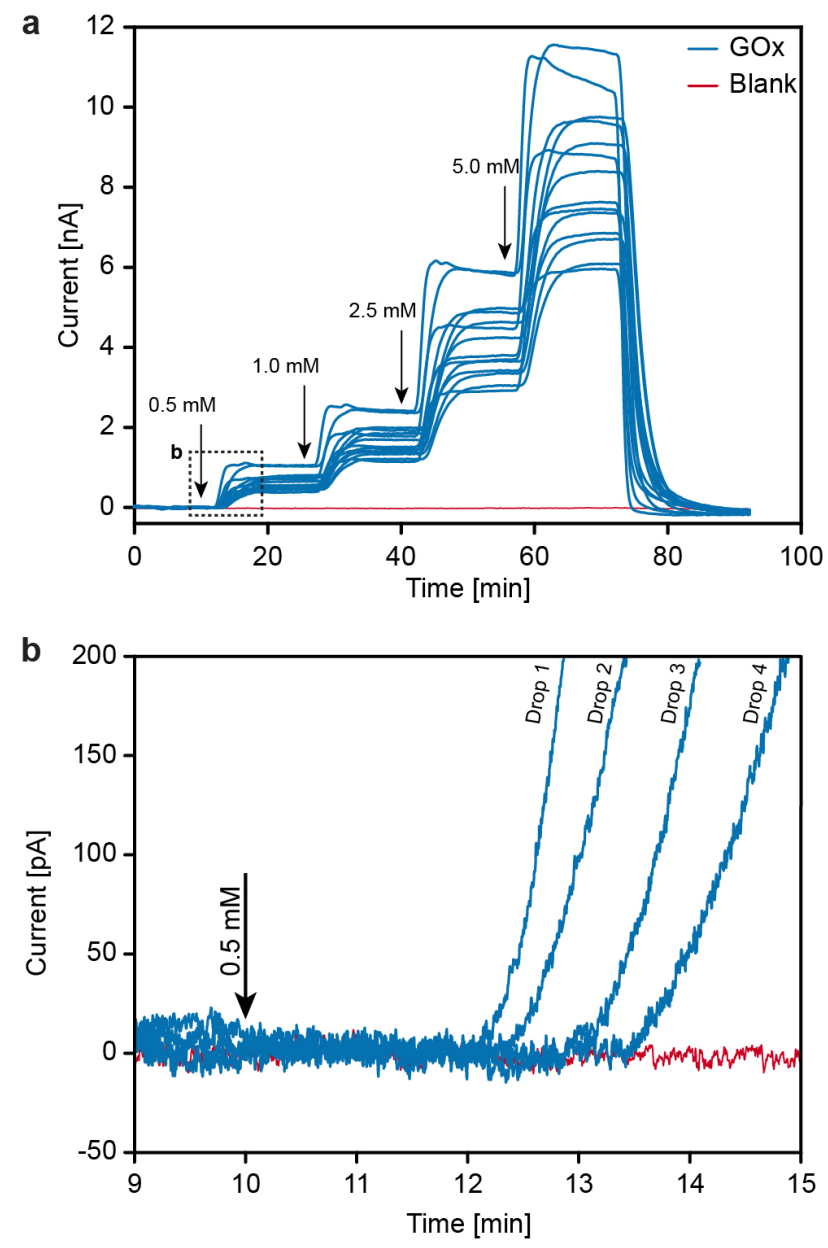

Figure 3: On-chip calibration of the glucose biosensors at $37{ }^{\circ} \mathrm{C}$. (a) Transient current signals of fourteen glucoseoxidase (GOx) functionalized electrodes (blue) and four electrodes coated with pure hydrogel (red). The sensor shows a linear response up to $5 \mathrm{mM}$ of glucose $(n=14)$. (b) The inset shows a zoomed-in view of the sensor response during the first glucose injection. The sequential delay of the response is due to the positions of the drops along the flow path along one branch in Fig. 1 (drops 1 - 4). 
In an open microfluidic system such as the hangingdrop network, an active in- and outflow is needed to perfuse medium through the system. Additionally, inflow and outflow rates need to be well controlled to maintain the drop sizes in the device. For longer experiments, evaporation effects could be observed and need to be compensated. This compensation requires very precise control of flow rates on both the syringe pumps that are connected to the inlets and of the peristaltic pump, which is used to withdraw the medium through the outlet port. Most of the conventional instruments cannot guarantee this degree of precision. To overcome this limitation and to compensate for evaporation, a feedback control was implemented to constantly fine-tune the inlet flow rate to ensure a stable drop size throughout an entire experiment. Therefore, a microscope was used to continuously monitor the position of the microtissues along the Z-axis in all hanging drops and a feedback algorithm automatically adjusted the inflow rate of the syringe pump.

The stability of the system operation based on drop height is shown in Figure 4. Drop heights were defined as the distance between the rim structure and the bottom of the hanging drop. The orange lines in the graph show the drop height of every individual drop, and the blue line shows the average drop height in the device. The average of the drop heights was stable between $630-700 \mu \mathrm{m}$.

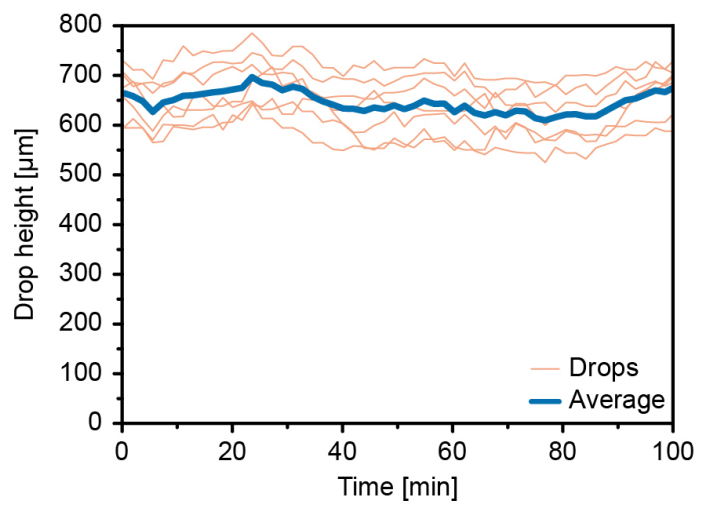

Figure 4: Drop heights during calibration of the device. A pump controller ensured controlled flow rates to stabilize drop heights throughout the experiment.

\section{CONCLUSION}

In this paper, we presented a novel approach for integrating microelectrode arrays into a microfluidic system. The technological approach takes advantage of all features of open microfluidic systems, such as bubble free operation and enhanced gas exchange and includes a versatile and scalable readout method. Simple microfabrication processes enable direct integration of electrode array and microfluidic guiding structures on the same substrate.

The presented results demonstrate the advantages of combining microfluidics for precise liquid handling with microelectrode arrays for parallel readout. The electrodes were functionalized for use as biosensors and enable real time detection of changes in glucose concentrations up to physiological concentrations. The biosensors showed spatially and temporally resolved response patterns upon changes in glucose content along the microfluidic flow path.

Hanging-drop networks were specifically developed for 3D cell culturing systems. The complete integration of biosensors in this platform allows for monitoring of the metabolism of 3D microtissues in situ [3]. Adding more electrodes, integration of other sensors, or expanding the fluidic network could increase the readout capability of such a device. Furthermore, the transparent glass substrate enables parallel microscopy readout, which is of interest for most biological applications and for continuous observation of the samples.

The presented platform has been specifically designed as an analytical cell culturing and monitoring tool to simultaneously observe the metabolic state of 3D microtissues.

\section{ACKNOWLEDGEMENTS}

This work was financially supported by FP7 of the EU through the through the ERC Advanced Grant "NeuroCMOS" (AdG 267351), the ICT-FET- 296257 project "Body on a chip", and an individual Ambizione Grant (Contract No. 142440) of the Swiss National Science Foundation for Olivier Frey.

\section{REFERENCES}

[1] F. Hirschhaeuser, H. Menne, C. Dittfeld, J. West, W. Mueller-Klieser, L.A. Kunz-Schughart, "Multicellular tumor spheroids: An underestimated tool is catching up again", Journal of Biotechnology, (2010) 148, 3-15.

[2] O. Frey, P.M. Misun, D.A. Fluri, J.G. Hengstler, A. Hierlemann, "Reconfigurable microfluidic hanging drop network for multi-tissue interaction and analysis", Nature Communications, 5:4250 (2014). doi: $10.1038 /$ ncomms 5250 .

[3] P.M. Misun, J. Rothe, Y.R.F. Schmid, A. Hierlemann, O. Frey, "Multi-analyte biosensor interface for realtime monitoring of 3D microtissue spheroids in hanging-drop networks", Microsystems and Nanoengineering, $\quad(2016) \quad 2, \quad 16022$. doi:10.1038/micronano.2016.22.

[4] G. Jobst, I. Moser, M. Varahram, P. Svasek, E. Aschauer, Z. Trajanoski, P. Wach, P. Kotanko, F. Skrabal, G. Urban, "Thin-film microbiosensors for glucose-lactate monitoring", Analytical Chemistry, (1996) vol. 68, pp. 3173-3179.

\section{CONTACT}

Patrick M. Misun,

tel: +41-61-387-33-70; patrick.misun@bsse.ethz.ch 